NASA Technical Memorandum 105169

AIAA-91-2264

\title{
Progress Toward Synergistic Hypermixing Nozzles
}

D.O. Davis and W.R. Hingst

Lewis Research Center

Cleveland, Ohio

Prepared for the

27th Joint Propulsion Conference

cosponsored by the AIAA, SAE, ASME, and ASEE

Sacramento, California, June 24-27, 1991

\section{N/5n}




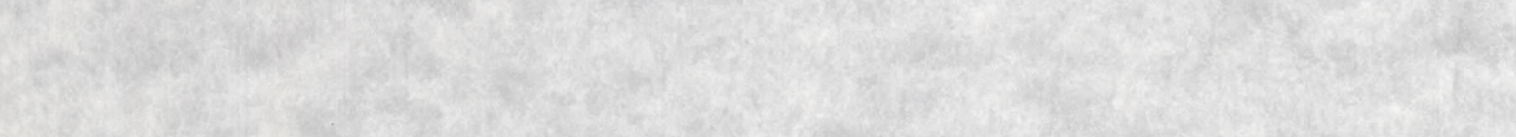

$(2+5=2 x$

as ${ }^{2}$

-

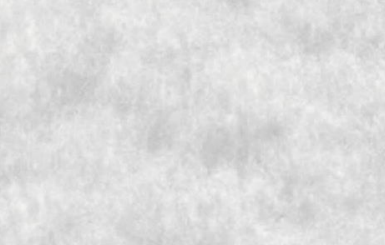

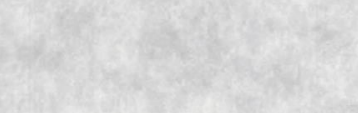

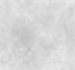

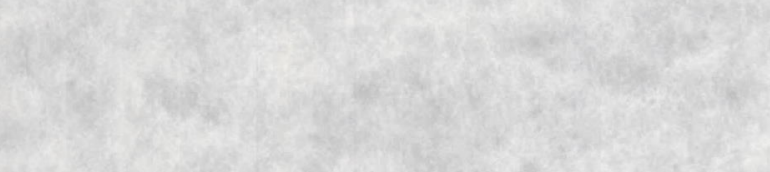

$3 x^{2}$

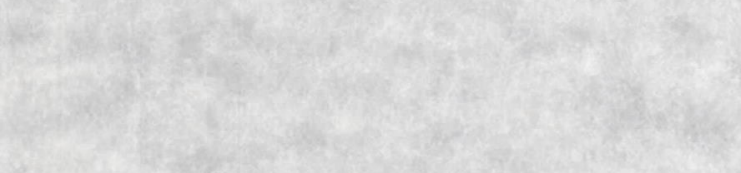

$3 x^{2}$

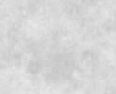

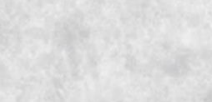
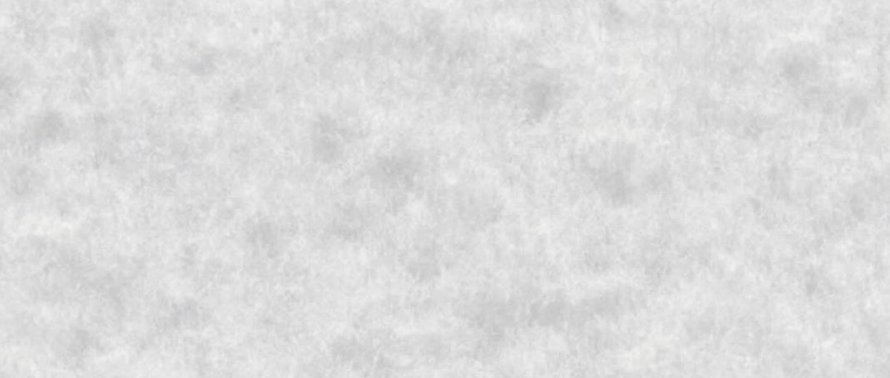

ax

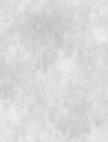

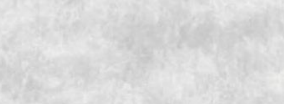

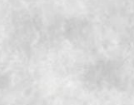

20

1093

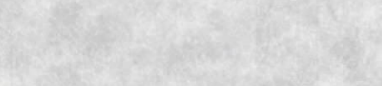

tax

(4)

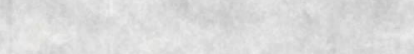

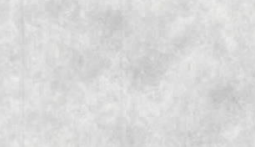




\title{
PROGRESS TOWARD SYNERGISTIC HYPERMIXING NOZZLES
}

\author{
D. O. Davis"and W. R. Hingst" \\ NASA Lewis Research Center, Cleveland, Ohio
}

\section{Abstract}

Mean flow measurements have been obtained for airto-air mixing downstream of swept and unswept ramp wall-mounted hypermixing nozzle configurations. Aside from the sweep of the ramps, the two nozzle configurations investigated are identical. The nozzles inject three parallel supersonic jets $\left(M_{j e t}=3.0\right)$ at a $15^{\circ}$ angle (relative to the wind tunnel wall) into a supersonic freestream. Mach number and volume fraction distributions in a transverse plane 11.1 nozzle heights downstream from the nozzle exit plane were measured. Data are presented for a freestream Mach number of three at a matched static pressure condition and also at an underexpanded static pressure condition (pressure ratio equal to five). Surface oil flow visualization was used to investigate the nearwall flow behavior. The results indicate that the swept ramp injectors produce stronger and larger vortex pairs than the unswept ramp injectors. The increased interaction between the swept ramp model's larger vortex pairs yields better mixing characteristics for this model.

\section{Nomenclature}

$\begin{array}{ll}D & =\text { width and height of wind tunnel } \\ H & =\text { nozzle height (Fig. } 2) \\ M & =\text { Mach number } \\ P & =\text { static pressure } \\ P_{t} & =\text { total pressure } \\ \bar{q} & =\text { dynamic pressure ratio }\left(\rho U^{2}\right)_{j} /\left(\rho U^{2}\right)_{\infty} \\ U & =\text { axial velocity component } \\ v & =\text { volume (mole) fraction } \\ x, y, z & =\text { cartesian coordinate system } \\ \lambda & =\text { mass flux ratio }(\rho U)_{j} /(\rho U)_{\infty} \\ \rho & =\text { density }\end{array}$

\section{Subscripts \\ $\mathrm{j}=$ jet exit condition \\ $\infty=$ wind tunnel freestream condition}

*Aerospace Engineer, Inlet, Duct, and Nozzle Flow Physics Branch, Member AIAA.

Copyright (C) 1991 by the American Institute of Acronautics and Astronautics, Inc. No copyright is asserted in the United States under Title 17, U.S. Code. The U.S. Government has a royalty-free license to exercise all rights under the copyright claimed herein for Govemmental purposes. All other rights are reserved by the copyright owner.

\section{Introduction}

$\mathbf{T}$ $\mathrm{HE}$ problem of complete fuel-oxidant mixing in SCRAMJET combustors, given the short residence time and the inherently low mixing rates, is not a trivial one. To obtain the necessary mixing rates, supersonic combustors will utilize wall-mounted and/or strutmounted injector nozzles, depending on combustor size. For wall-mounted injectors, the angle of injection is an important consideration. Normal injection provides good mixing, but also high pressure loss due to the normal bow shock. Parallel slot injection contributes all of it's momentum to axial thrust, but at the expense of poor mixing and no penetration. Mays et al. ${ }^{1}$ have shown that for flush nozzles, penetration nearly equivalent to normal injection can be achieved with low angle injectors. If a low angle injector is ramped to create a protrusion into the flow, then skew-induced streamwise vorticity is generated which enhances the mixing process. In addition, the oblique shock wave generated by the ramp will reflect from the opposing wall and cross the injector stream. There is evidence that this interaction will also increase mixing. ${ }^{2}$ For these reasons, ramped injector nozzles ("hypermixing nozzles") are a particularly attractive method to enhance high-speed mixing.

To date, nearly all of the studies of wall-mounted hypermixing nozzles have been based on the swept and unswept ramp models developed at NASA Langley. ${ }^{3}$ These models have been the subject of numerical studies for reacting hydrogen-air mixing ${ }^{4,5,6}$ and non-reacting hydrogen-air mixing ${ }^{4,5,6,7}$ and of experimental studies for reacting and non-reacting hydrogen-air mixing ${ }^{3}$ and non-reacting air-air mixing. ${ }^{8}$ These studies have shown that the swept ramp model provides a higher degree of mixing, but at the expense of higher pressure losses, when compared with the unswept ramp model. In a related study, Hung and Barth ${ }^{9}$ performed a numerical study of hypersonic flow in the expansion slot of a generic unswept ramp injector configuration.

An experimental study is underway at NASA Lewis Research Center to investigate the effectiveness of wallmounted hypermixing nozzle concepts for high-speed propulsion applications. A swept and an unswept ramp model which are similar, yet different, to the Langley models are being tested. The objective of these tests is to provide insight into jet penetration and mixing behavior for a wide range of operating conditions, as well as to provide sufficiently detailed measurements in the crossplane to support CFD code calibration/validation efforts. 


\section{Experimental Program}

\section{Hypermixing Nozzles}

The nozzle configurations investigated are illustrated in Fig. 1. The two configurations are identical with the exception of the injector ramps; one has straight ramps, "Unswept Ramp Model (URM)," and one has swept ramps, "Swept Ramp Model (SRM)." The overall dimensions of the models are $30.5 \times 30.5 \times 5.37 \mathrm{~cm}$. The compression and expansion ramps are at a $10^{\circ}$ angle relative to the wind tunnel wall. The Mach number of the jets is nominally 3 and the injection angle of the jets is $15^{\circ}$. The dimensions of the jet exit are $2.54 \times 5.37$ $\mathrm{cm}$ and the spacing between the jets is $7.62 \mathrm{~cm}$. The nozzles form a knife edge at the exit plane to simplify mesh generation for computational purposes.

\section{Experimental Facility}

The experiments were performed in the NASA Lewis Research Center $1 \times 1$ (ft.) Supersonic Wind Tunnel, a continuous flow facility where the Mach number is varied by interchangeable nozzle blocks. A schematic of the wind tunnel test section for the present hypermixing experiments is shown in Fig. 2. The models span the full width of the wind tunnel. Total and static pressure, as well as trace gas sampling measurements, were taken in a plane 16.8 nozzle heights downstream from the leading edge of the compression ramp. For these models, the upstream boundary layer thickness is approximately half the

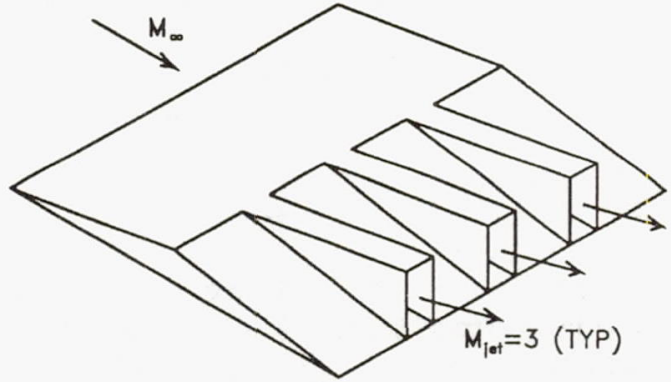

a) Unswept Ramp Model (URM).

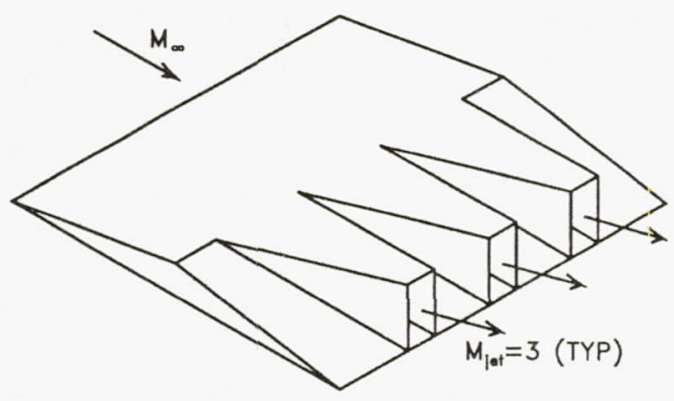

b) Swept Ramp Model (SRM).

Fig. 1 Hypermixing nozzle configurations.

maximum height of the nozzles. The models are mounted on a wall of the wind tunnel which corresponds to a contoured wall of the wind tunnel Laval nozzle. This was necessary to avoid the distorted boundary layer which is

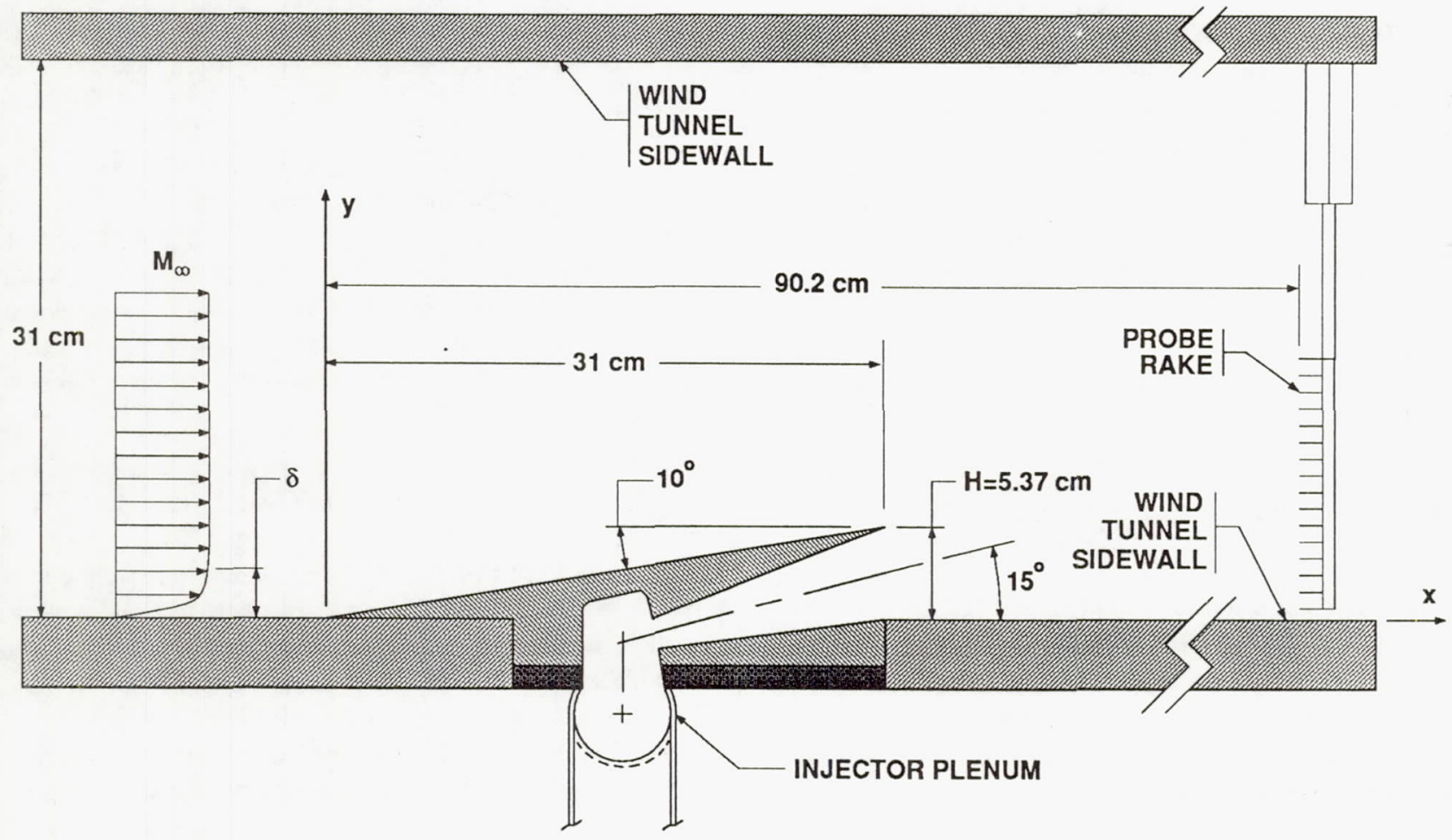

Fig. 2 Wind tunnel schematic. 
known to develop along the non-contoured walls of the Laval nozzle.

\section{Instrumentation}

Surface oil flow visualization was used as an aid to determine shock wave impingement location and local flow separation. The oil flow pattern also gives an indication of limiting wall streamline behavior and a relative indication of surface shear stress; regions where oil is scrubbed from the surface is indicative of high surface shear and regions of heavy oil accumulation indicates low surface shear. Pitot and static pressure measurements, from which the Mach number is computed, are taken in the crossplane for a number of operating conditions. From these results, several conditions were selected to study mixing by a hydrocarbon trace gas technique. Briefly, the trace gas technique involves seeding the injector flow with ethylene to a concentration of approximately $250 \mathrm{ppm}$. The flow is sampled with a Pitot tube in the measurement plane and analyzed with a flame ionization detector (FID) hydrocarbon analyzer. Normalizing the concentration in the measurement plane by the initial injected concentration yields the volume fraction of jet fluid. For the present results, mass fraction and volume fraction are equivalent.

\section{Results and Discussion}

\section{Operating Conditions}

The operating conditions for the hypermixing models are summarized in Table 1. Data were accumulated for freestream Mach numbers of 2, 3 and 4, however, only data at Mach 3 will be presented in this paper. At each Mach number, several jet total pressures were investigated. Trace gas measurements are indicated by the superscripts on the case numbers. All measurements were performed for nearly matched or underexpanded static pressure conditions. In addition, for each freestream Mach number, Pitot and static pressure surveys were obtained for the case when the nozzle flow was turned off. This helps distinguish between geometry induced and jet induced features of the flowfield. The reduced freestream total pressure for Case $2 \mathrm{a}$ was the result of a temporary limitation on wind tunnel mass flow due to equipment failure.

Inviscid oblique shock wave trajectories relative to the measurement plane for the three freestream Mach numbers are shown in Fig. 3. For $M_{\infty}=2$, the wave reflects from the opposing wall and impinges on the nozzle wall upstream of the measurement plane. At this condition, it is likely that the wave separates the boundary layer on the opposing wall, which would alter the reflection angle. For $M_{\infty}=3$, the wave reflects from the opposing wall and then crosses the measurement plane. For $M_{\infty}=4$, the reflection occurs downstream of the measurement plane.

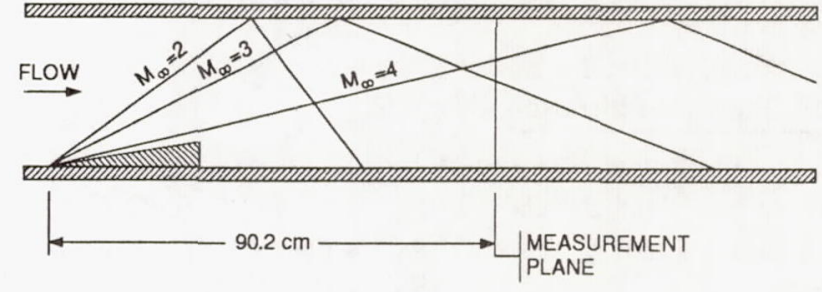

Fig. 3 Inviscid shock wave trajectories.

Table 1 Operating conditions.

\begin{tabular}{|cccccccc|}
\hline Case & $\mathbf{M}_{\infty}$ & $\mathbf{P}_{\mathbf{t}, \infty}(\mathrm{kPa})$ & $\mathbf{P}_{\mathbf{t}, \mathrm{j}} / \mathbf{P}_{\mathbf{t}, \infty}$ & $\mathbf{P}_{\mathbf{j}} / \mathbf{P}_{\infty}$ & $\mathrm{U}_{\mathbf{j}} / \mathbf{U}_{\infty}$ & $\lambda$ & $\overline{\mathbf{q}}$ \\
\hline $1^{\dagger}$ & 2.0 & 103.4 & 5.00 & 1.07 & 1.20 & 1.99 & 2.40 \\
2 & 2.0 & 103.4 & 10.0 & 2.13 & 1.20 & 3.99 & 4.79 \\
$2 \mathrm{a}^{* \dagger}$ & 2.0 & 86.2 & 12.0 & 2.56 & 1.20 & 4.78 & 5.75 \\
3 & 2.0 & 103.4 & 13.3 & 2.84 & 1.20 & 5.31 & 6.39 \\
$4^{* \dagger}$ & 3.0 & 206.9 & 1.00 & 1.00 & 1.00 & 1.00 & 1.00 \\
5 & 3.0 & 206.9 & 1.50 & 1.50 & 1.00 & 1.50 & 1.50 \\
6 & 3.0 & 206.9 & 2.00 & 2.00 & 1.00 & 2.00 & 2.00 \\
7 & 3.0 & 206.9 & 3.33 & 3.33 & 1.00 & 3.33 & 3.33 \\
$8^{* \dagger}$ & 3.0 & 206.9 & 5.00 & 5.00 & 1.00 & 5.00 & 5.00 \\
9 & 4.0 & 275.8 & 0.75 & 3.10 & 0.92 & 1.90 & 1.74 \\
10 & 4.0 & 275.8 & 1.12 & 4.65 & 0.92 & 2.85 & 2.62 \\
$11^{\dagger}$ & 4.0 & 275.8 & 1.50 & 6.20 & 0.92 & 3.80 & 3.49 \\
12 & 4.0 & 275.8 & 1.88 & 7.75 & 0.92 & 4.75 & 4.36 \\
13 & 4.0 & 275.8 & 2.50 & 10.33 & 0.92 & 6.33 & 5.81 \\
\hline
\end{tabular}

* Trace gas measurements URM, † Trace gas measurements SRM 


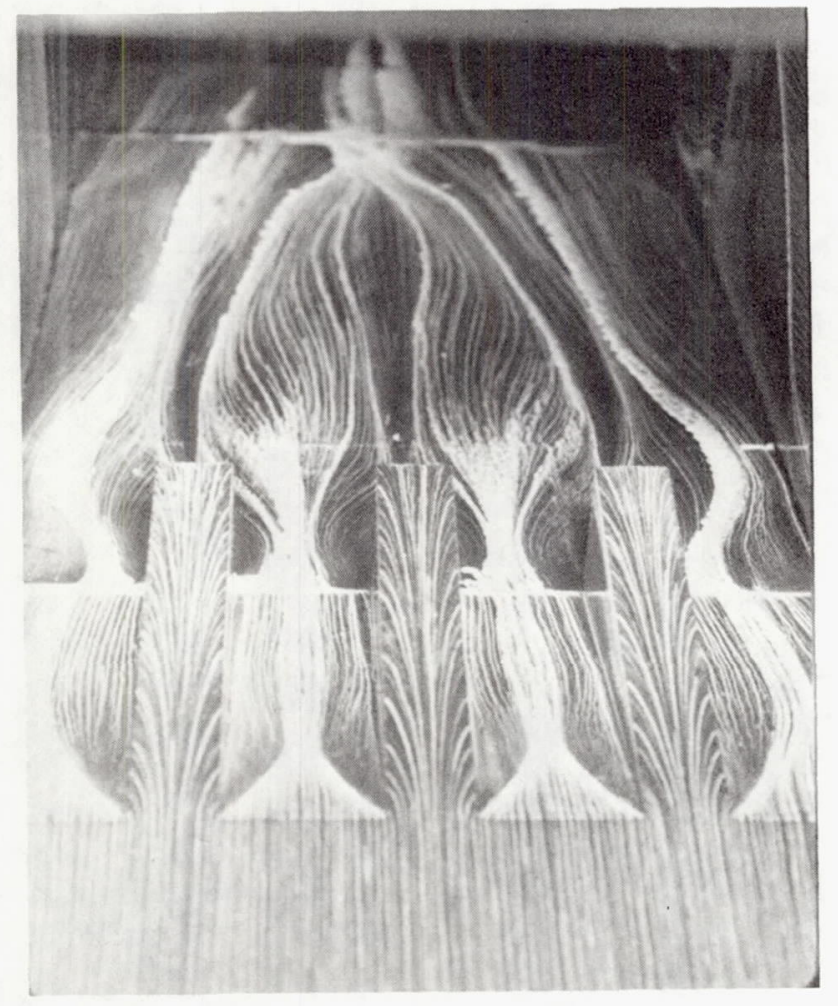

Fig. 4 Oil flow pattern for URM, Case 7.

\section{Oil Flow Visualization}

Oil flow visualization for the unswept and swept ramp models at the Case 7 operating condition are shown in Figs. 4 and 5, respectively. Although the views are shown looking down the wind tunnel floor, the models were actually mounted on a vertical wall of the wind tunnel which caused regions of heavy oil accumulation to sag slightly (to the right in the figures) after the wind tunnel was shutdown. Aside from this, the near-wall flow exhibits excellent symmetry. The different models qualitatively exhibit similar behavior. The converging of the near-wall flow towards the centerline indicates that a strong sidewall interaction is present. The rate of convergence was observed to be proportional to the jet total pressure. No flow separation is evident for either model at this operating condition. For the cases where $M_{\infty}=3$ and 4 , the bow shock which reflects from the opposing wall impinges on the model wall downstream of the measurement plane (refer to Fig. 3). For $M_{\infty}=2$, however, the reflected bow shock impinges upstream of the measurement plane and oil flow patterns for this case (not shown) clearly indicate a symmetric three-dimensional flow separation.

\section{Mach Number Contours}

Mach number distributions in the crossplane were computed from Pitot and static pressure surveys. These pressure measurements were made on a rectangular grid with a cell size of $6.35 \times 6.35 \mathrm{~mm}$. For these plots, the

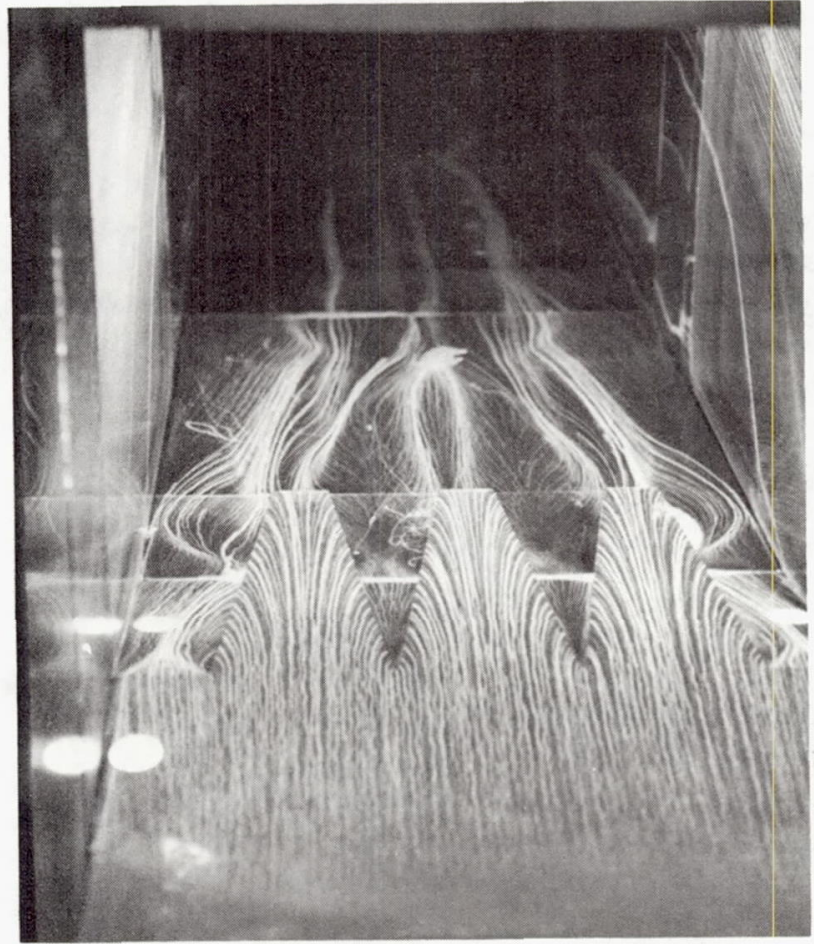

Fig. 5 Oil flow pattern for SRM, Case 7.

Pitot and static pressure were measured in the range $0<$ $\mathrm{y} / \mathrm{D}<0.5$ and $-0.5<\mathrm{z} / \mathrm{D}<0.3$, where $\mathrm{D}$ is the height and width of the wind tunnel. Although a plane of symmetry exists at $\mathrm{z} / \mathrm{D}=0$, the measurements were extended into the right duct half in order to verify spanwise symmetry of the flowfield.

Mach number distributions were measured for the case when the jet flow was turned off to determine the effect that the nozzle geometry alone has on the flowfield. Mach number contours for a freestream Mach number of three are shown for the URM and SRM in Figs. 6 and 7, respectively. The axes of these and subsequent plots correspond to the wind tunnel walls. The outward bulging of the Mach contours in the vicinity of $y / D=0.5, z / D=-0.4$ is a result of the streamwise vortex pair generated by uneven expansion of the near-wall flow on the straight walls of the wind tunnel Laval nozzle. Symmetry about the horizontal midplane $(\mathrm{z} / \mathrm{D}=0)$ within the region -0.3 $<\mathrm{z} / \mathrm{D}<0.3$ is seen to be very good. The results show that the different model geometries create significantly different flowfields. For the URM (Fig. 6), the flowfield directly in line with the individual injectors is characterized by the horseshoe shaped contours resulting from vortex pairs with the common flow away from the nozzle wall. The contour pattern for each injector is roughly of the same size and shape indicating limited interaction between the vortex pairs. For the SRM (Fig. 7), the contour patterns indicate larger vortex pairs that interact with each other. This concurs with the computational results obtained by Moon $^{6}$ of a comparison between the NASA 


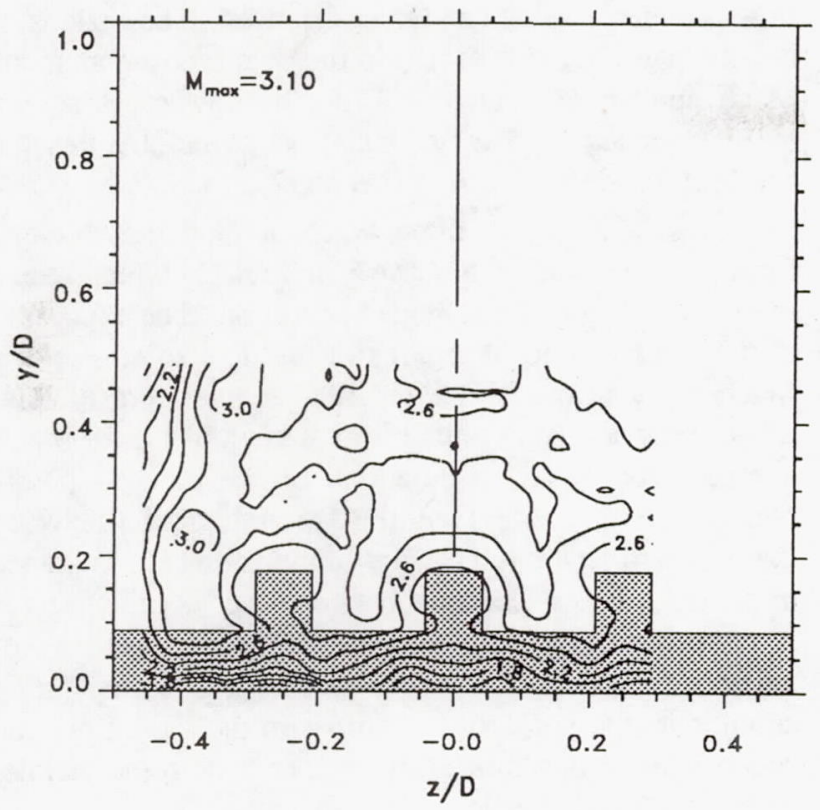

Fig. 6 Mach number contours

for $U R M, M_{\infty}=3, M_{j}=0$.

Langely unswept and swept ramp models. Moon's results showed that the swept ramp model's jet spread more rapidly and had nearly twice the level of streamwise vorticity as the unswept model. The higher vorticity levels associated with the swept ramp model causes increased flow losses. This can be seen for the present configuration by comparing Mach number levels in the

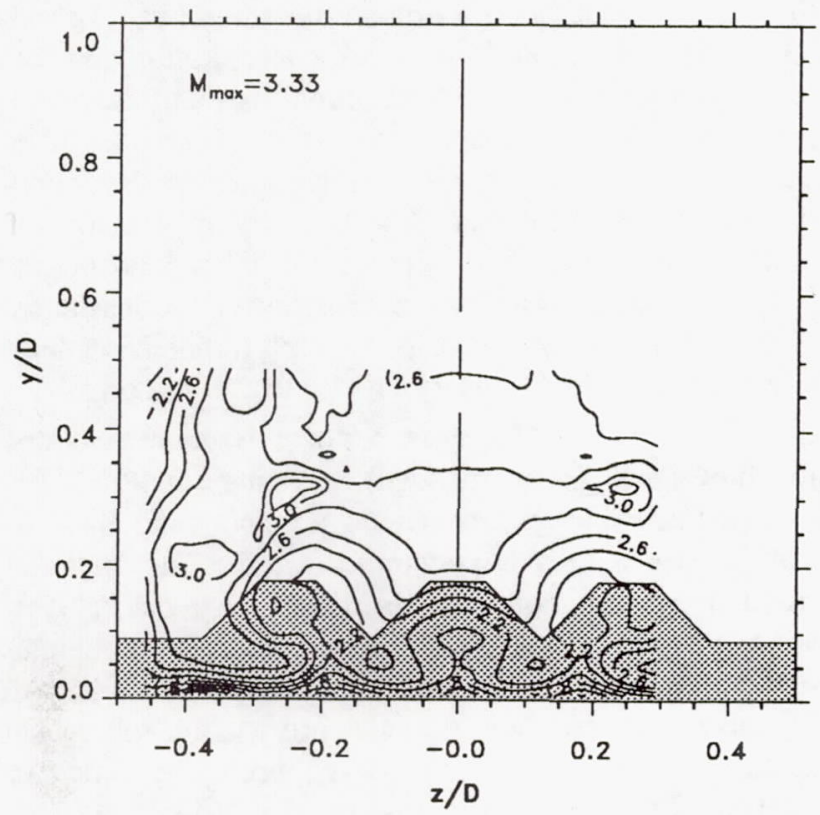

Fig. 7 Mach number contours for $\mathrm{SRM}, \mathrm{M}_{\infty}=3, \mathrm{M}_{\mathrm{j}}=0$.

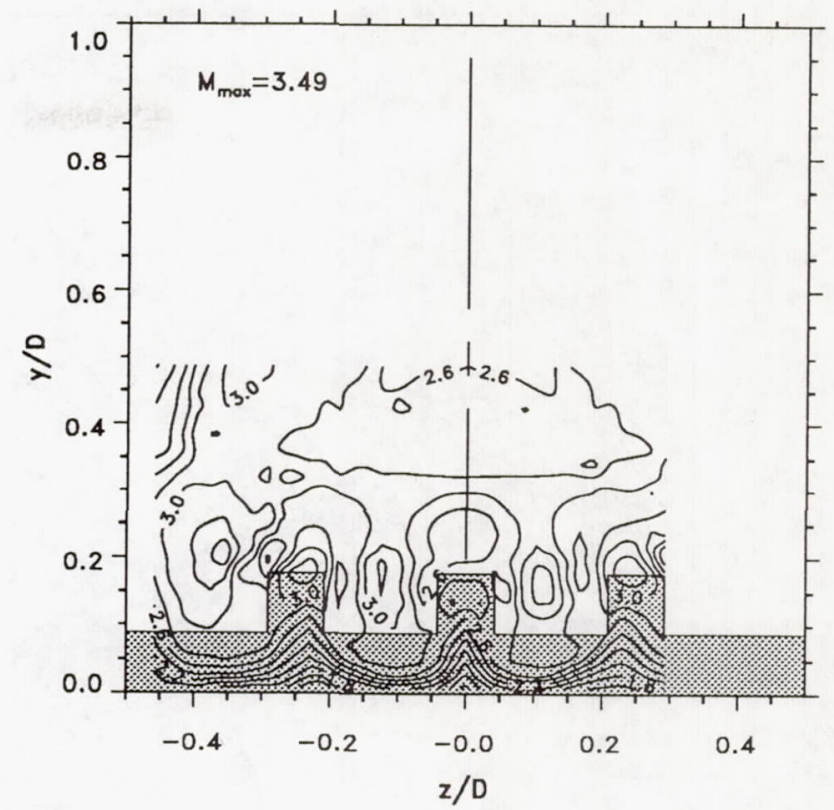

Fig. 8 Mach number contours for URM, $M_{\infty}=3, \bar{q}=1$ (Case 4).

vicinity of the vortex pairs. With reference to Fig. 6 , the 2.2 Mach number contour for the URM is observed to be nearly straight and running parallel to the nozzle wall. For the SRM (Fig. 7), the 2.2 level bulges up into the core of the vortex pairs. The causes of the higher losses are two fold. First, more axial momentum is lost in the conversion to angular momentum as the stronger

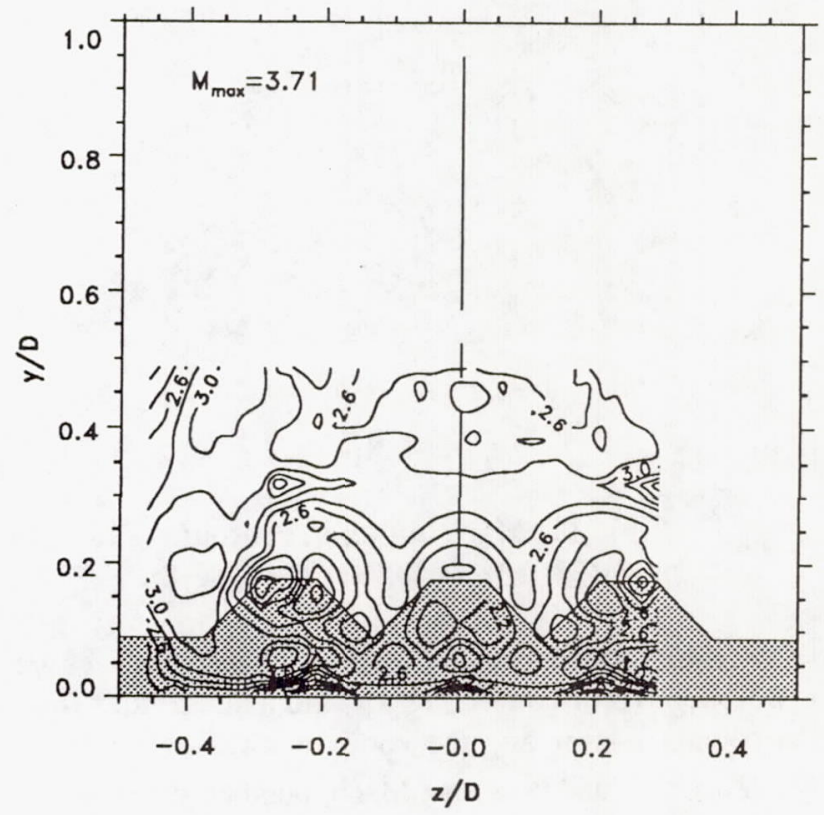

Fig. 9 Mach number contours for SRM, $M_{\infty}=3, \bar{q}=1$ (Case 4). 


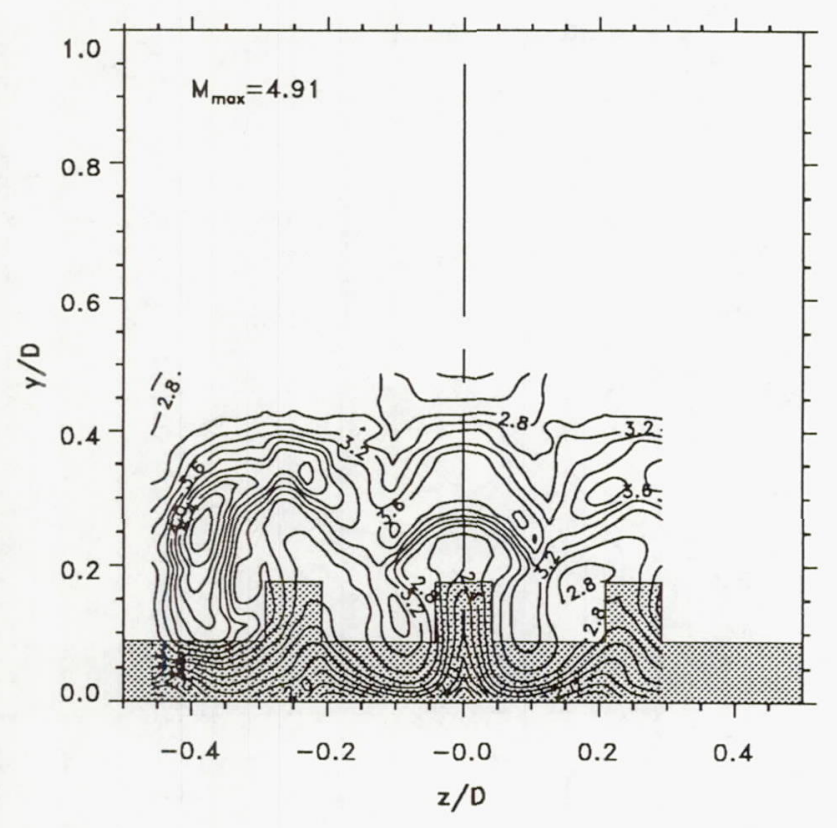

Fig. 10 Mach number contours

for URM, $M_{\infty}=3, \bar{q}=5$ (Case 8).

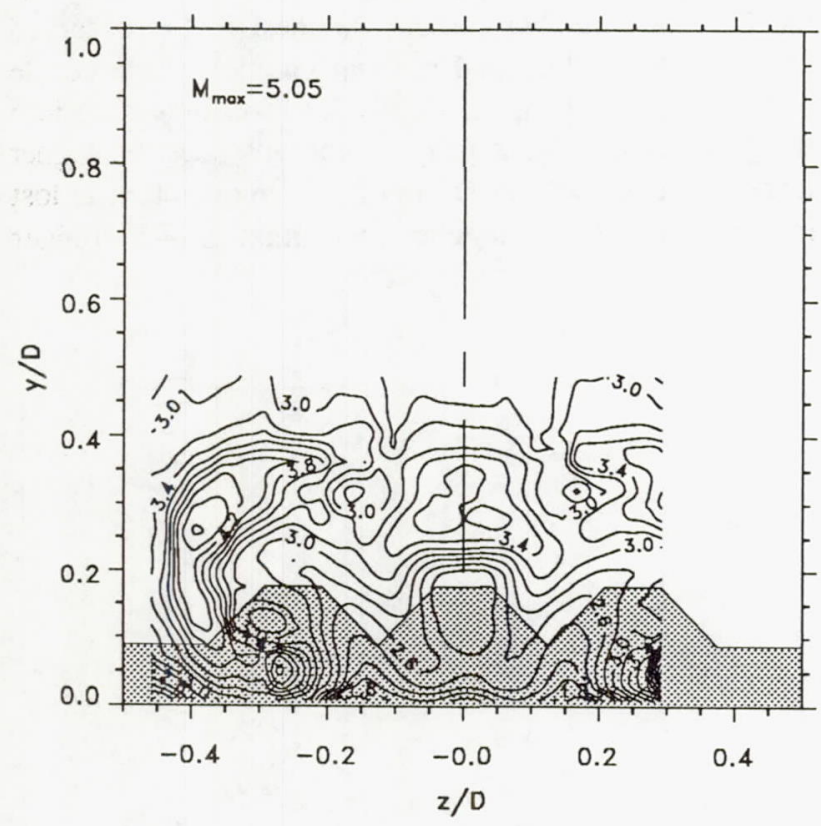

Fig. 11 Mach number contours for SRM, $M_{\infty}=3, \bar{q}=5$ (Case 8).

vortices are formed. And secondly, the larger vortices are more effective in convecting low-momentum fluid from the boundary layer into the vortex cores.

Figs. 8 and 9 show Mach number distributions for the Case 4 operating condition. For this case, the jet exit condition and the upstream wind tunnel core condition were exactly matched. At the nozzle exits, however, there are likely to be local regions where the flow is underexpanded and overexpanded. The regions of underexpansion are reflected in the increase in maximum Mach number compared with the no-flow cases shown in Figs. 6 and 7. Again, spanwise symmetry for this condition is observed to be very good.

Figs. 10 and 11 show Mach number distributions for a pressure ratio of 5 (Case 8 in Table 1) which represents a strongly underexpanded condition. The expansion of the jets cause local regions of the flow to approach a Mach 5 condition. Since the total temperature of both the jet flow and wind tunnel flow was ambient, for Mach numbers above 4.5 , liquefaction of the air was possible. At this operating condition, the interaction between the outboard jets and the wind tunnel sidewalls becomes important. The interaction between the jets themselves becomes greater for both models, but it is still more pronounced for the SRM. As with the previous two operating conditions, the peak Mach number in the vicinity of the center injector is significantly smaller than in the vicinity of the outboard injectors.

\section{Volume Fraction Contours}

The trace gas measurements were obtained on the same rectangular grid as the pressure measurements. However, because of the relatively long time that it takes to obtain a complete trace gas survey (each sample takes about 25 seconds), symmetry was assumed about the $\mathrm{z} / \mathrm{D}$ $=0$ plane, so that measurements were only taken in one duct half. For presentation purposes, in the following plots the data has been mirror imaged about the vertical centerline to show the entire wind tunnel.

Volume fraction contours for the URM and SRM at the Case 4 operating condition are shown in Figs. 12 and 13 , respectively. The lower peak concentration and the larger mixed area for the SRM confirms that this model provides better mixing than the URM. These plots also show the deeper penetration into the boundary layer and the higher degree of interaction between the jets for the SRM. From a mixing standpoint, the interaction of the vortices with the boundary layer is beneficial, inasmuch as the secondary motion convects high turbulence intensity fluid from the boundary layer into the jet cores.

Figs. 14 and 15 show volume fraction contours for the Case 8 (underexpanded) operating condition. As expected, the increased dynamic pressure ratio for this case causes a greater penetration of the jets into the freestream. For both models, there are local regions where the jet fluid is essentially unmixed. These regions of unmixed fluid are generally larger for the URM. At this operating condition, the outboard jets are rolling up under the center jet. This roll-up behavior, which is evident in the surface oil flow patterns shown in Figs. 4 and 5, is more pronounced for the SRM and is a result of higher streamwise vorticity levels generated by this model geometry. 


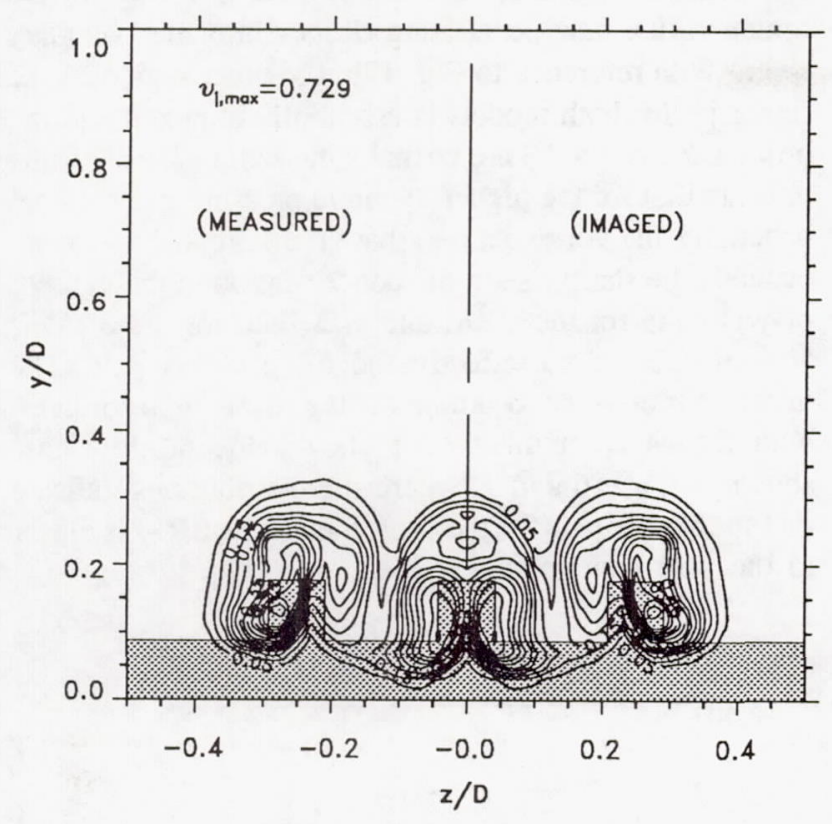

Fig. 12 Volume fraction contours for URM, $M_{\infty}=3, \bar{q}=1$ (Case 4).

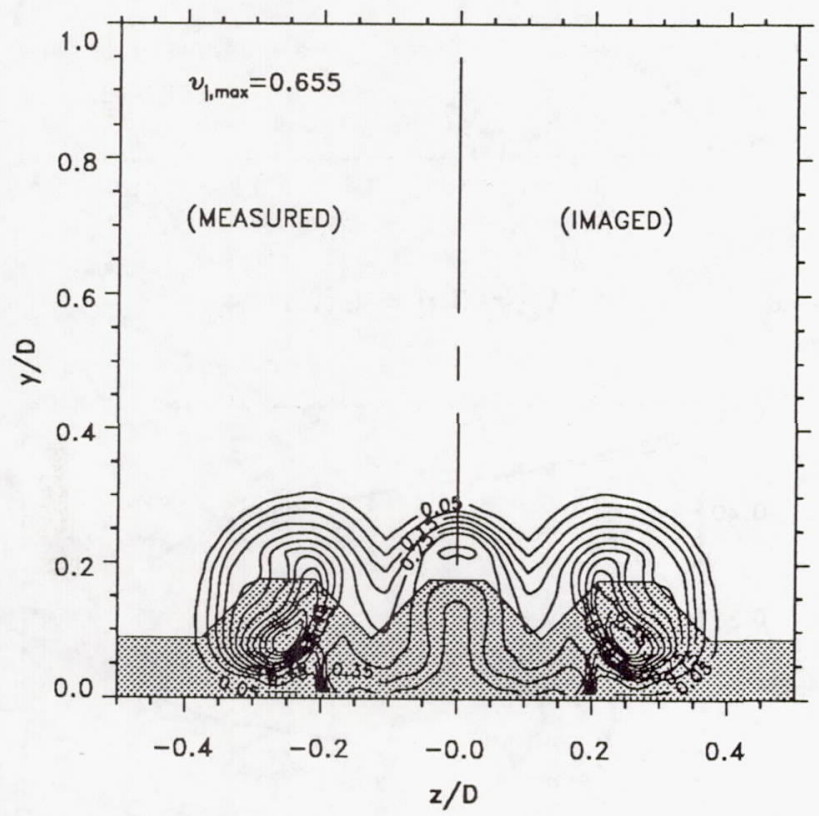

Fig. 13 Volume fraction contours for $S R M, M_{\infty}=3, \bar{q}=1$ (Case 4).

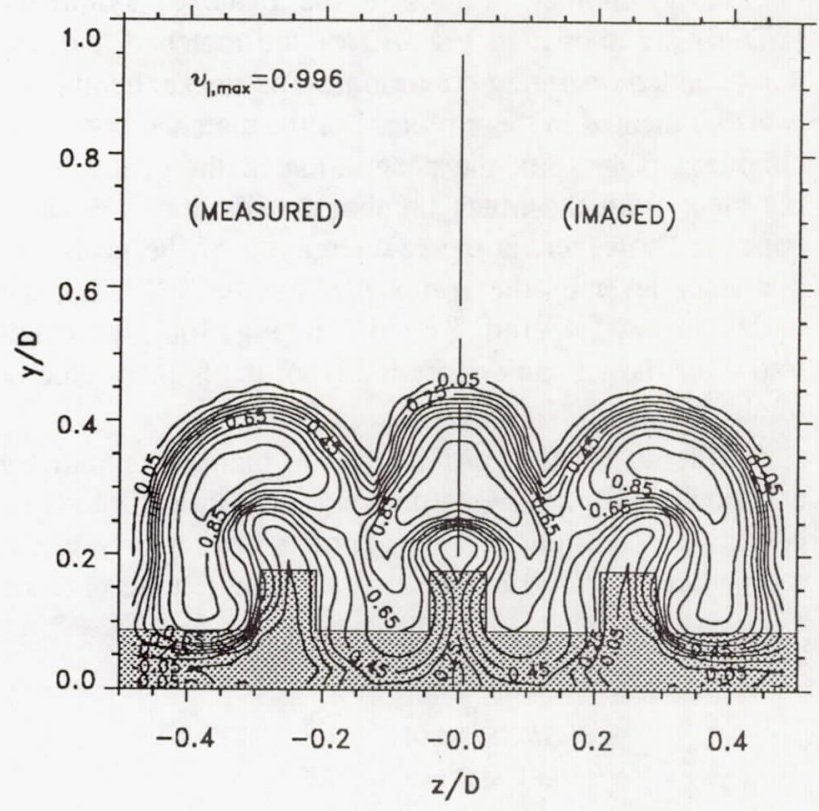

Fig. 14 Volume fraction contours for URM, $M_{\infty}=3, \bar{q}=5$ (Case 8).

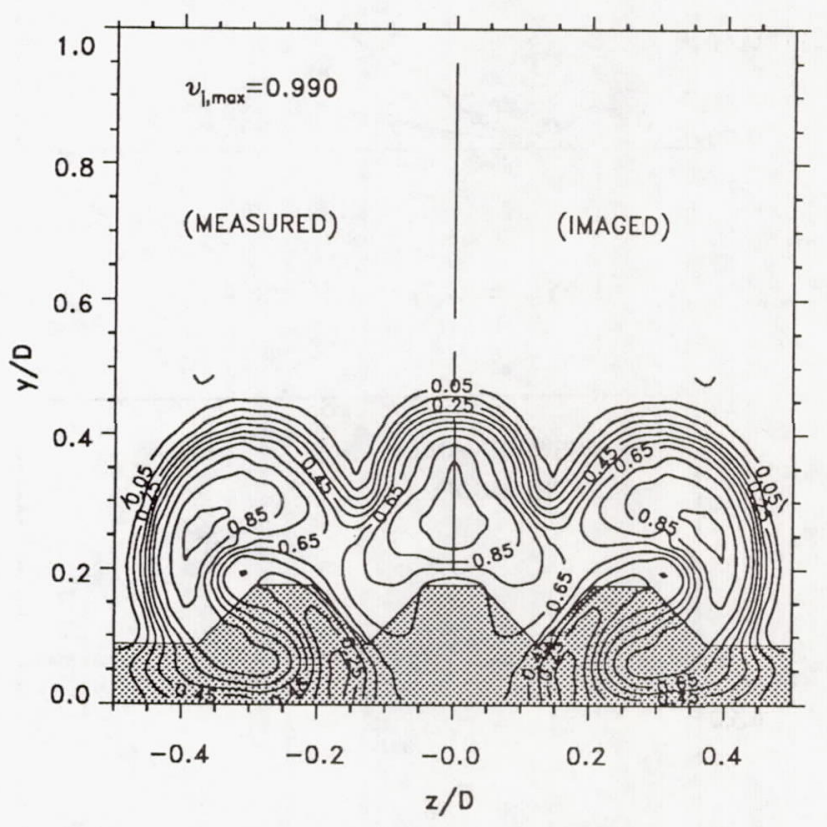

Fig. 15 Volume fraction contours for $S R M, M_{\infty}=3, \bar{q}=5$ (Case 8 ). 


\section{Profiles in the Plane of Symmetry}

Mach number profiles in the plane of symmetry $(\mathrm{z} / \mathrm{D}=0)$ are shown in Fig. 16 for the matched and underexpanded operating conditions. The nozzle height $(\mathrm{H})$ is also indicated in these plots. For the matched operating condition (Fig. 16a), the penetration of the center jet is not clear from the Mach number distributions. What is apparent, however, is the re-energizing of the near-wall boundary layer by the vortex pair for the SRM. For the underexpanded operating condition (Fig. 16b), the penetration of the jet can be inferred from the higher velocity of the jet fluid.

Volume fraction profiles in the plane of symmetry are shown in Fig. 17. Qualitatively, the results for the two operating conditions are very similar, particularly in the region below $y / D=0.2$. The most striking feature of these

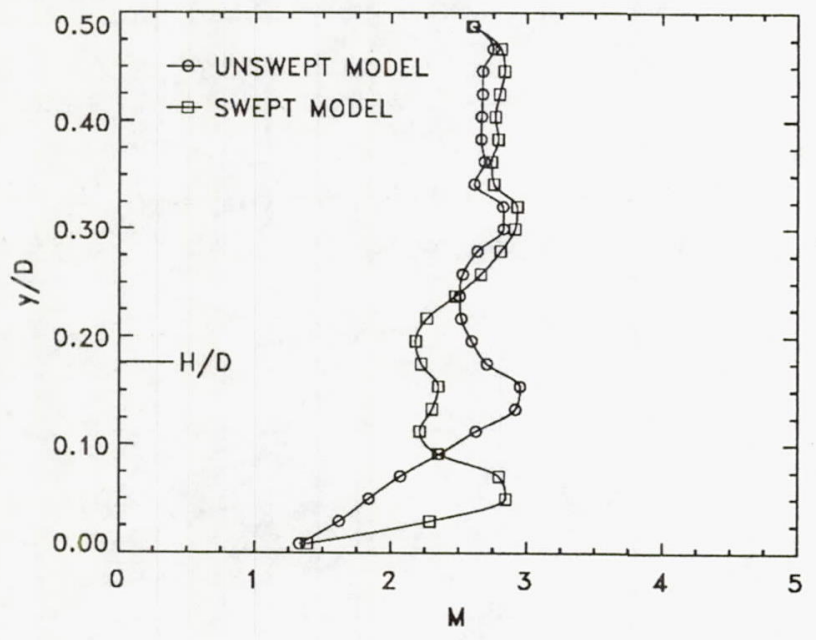

a) $\mathrm{M}_{\infty}=3, \overline{\mathrm{q}}=1$ (Case 4$)$.

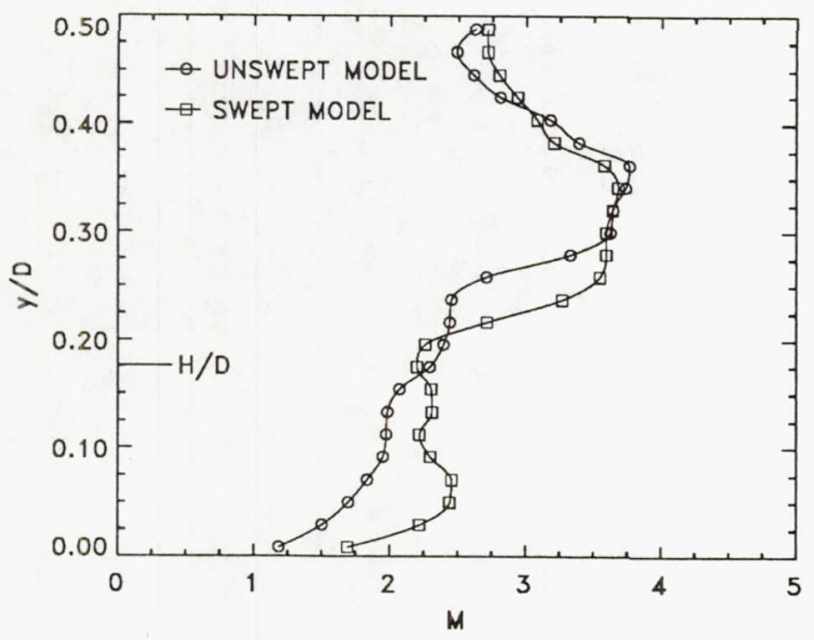

b) $\mathrm{M}_{\infty}=3, \overline{\mathrm{q}}=5$ (Case 8$)$.

Fig. 16 Mach number profiles in the plane of symmetry $(\mathrm{z} / \mathrm{D}=0)$. results is the relatively high concentration of jet fluid in the near-wall region for the SRM which is a result of the center vortex pair penetrating deeper into the boundary layer. With reference to Fig. $17 \mathrm{~b}$, the outer region of the center jet for both models is essentially unmixed and the profiles for $y / D>0.3$ are virtually the same. These results indicate that for the higher dynamic pressure ratio, the jet penetrates the vortex pair so that no mixing enhancement occurs. The sharp rise in the concentration in the vicinity of $y / D=0.25$ for the URM and $y / D=0.20$ for the SRM is an indication of the effective edge the vortex pair. The different transverse locations for the rise is in accordance with the Mach number contours for the no-flow case shown in Figs. 6 and 7, where the distributions indlicate that the vortex pair for the center jet of the SRM is closer to the wall than for the URM.

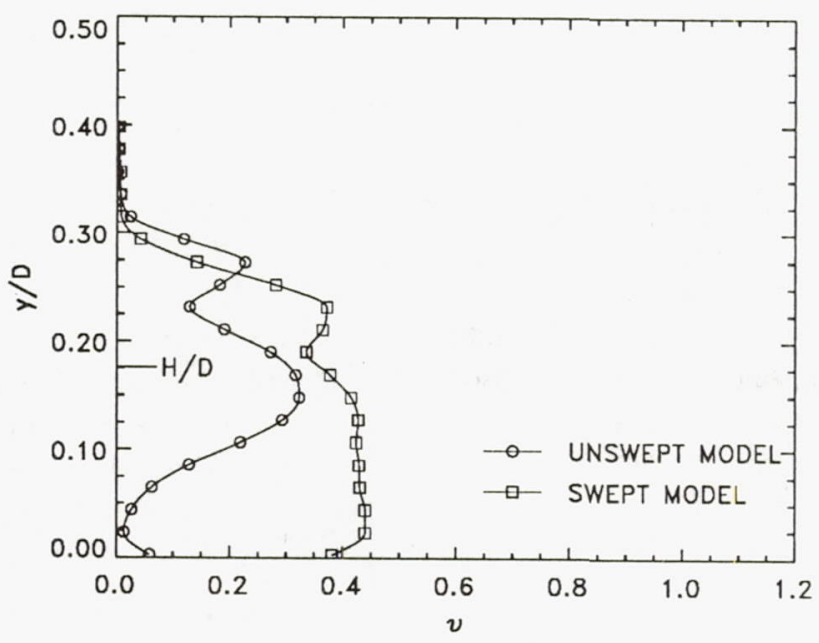

a) $\mathrm{M}_{\infty}=3, \overline{\mathrm{q}}=1$ (Case 4$)$.

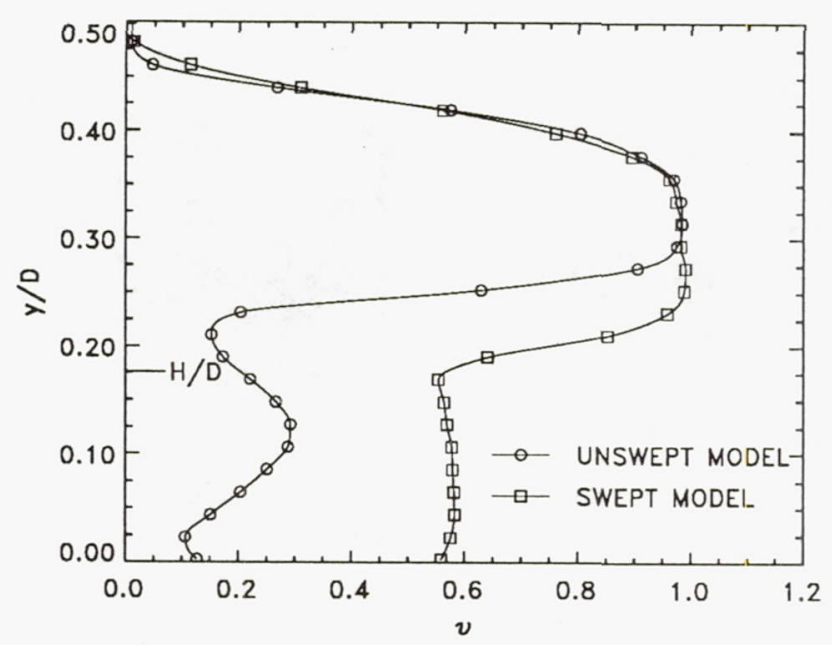

b) $\mathrm{M}_{\infty}=3, \overline{\mathrm{q}}=5$ (Case 8 ).

Fig. 17 Volume fraction profiles in the plane of symmetry $(z / D=0)$. 
Representative results of the hypermixing tests being conducted at NASA Lewis have been presented. The results for the present hypermixing configurations can be summarized as follows. The Mach number and volume fraction distributions indicate that the swept ramp model produces vortex pairs which are stronger and larger than those produced by the unswept ramp model. Inasmuch as the spanwise spacing of the injectors is the same for both models, the larger vortex pairs associated with the SRM have a higher degree of interaction. The interaction between the vortex pairs and with the boundary layer cause the center jet of the swept ramp model to mix faster than the outboard jets. Higher flow losses, however, are observed for the swept ramp model. Increasing the jet total pressure has two effects. First, interaction with the sidewalls of the wind tunnel causes the near wall flow to converge toward the centerline of the wind tunnel. Secondly, the increased dynamic pressure causes the upper half of the jet to penetrate beyond the influence of the vortex pair, resulting in low mixing rates.

The first phase of testing, which has been the subject of this paper, has been completed. The data is currently being further analyzed to quantify flow losses and mixing efficiencies. From the results obtained thus far, one or two operating conditions will be selected for further study. The next phase of testing will involve more detailed measurements at the selected operating conditions which will include measurements at more axial stations, mean transverse velocity measurements by means of a five-hole pressure probe and turbulence quantities measured by means of hot-wire techniques.
${ }^{1}$ Mays, R. B., Thomas, R. H., and Schetz, J. A., "Low Angle Injection Into a Supersonic Flow," AIAA Paper 89-2461, 1989.

${ }^{2}$ Menon, S., "Shock Waves for Enhanced Mixing in Scramjet Combustors," NASA NASP CR 1028, Dec. 1988.

${ }^{3}$ Northam, G. B., Greenburg, I., and Byington, C. S., "Evaluation of Parallel Injector Configurations for Supersonic Combustion," AIAA Paper 89-2525, July 1989.

${ }^{4}$ Riggins, D. W., Mekkes, G. L., McClinton, C. R., and Drummond, J. P., "A Numerical Study of Mixing Enhancement in a Supersonic Combustor," AIAA Paper 90-0203, Jan. 1990.

${ }^{5}$ Riggins, D. W. and McClinton, C. R., "A Computational Investigation of Flow Losses in a Supersonic Combustor," AIAA Paper 90-2093, July 1990.

${ }^{6}$ Moon, Y., "Numerical Study of Supersonic Combustors by Multi-Block Grids with Mismatched Interfaces," AIAA Paper 90-5204, 1990.

${ }^{7}$ Drummond, J. P., Carpenter, M. H., Riggins, D. W., and Adams, M. S., "Mixing Enhancement in a Supersonic Combustor," NASA NASP TM 1104, May 1990.

${ }^{8}$ Hartfield, R. J., Hollo, S. D., and McDaniel, J. C., "Experimental Investigation of a Supersonic Swept Ramp Injector Using Laser-Induced Iodine Fluorescence," AIAA Paper 90-1518, 1990.

${ }^{9}$ Hung, C. M. and Barth, T. J., "Computation of Hypersonic Flow Through a Narrow Expansion Slot," AIAA Paper 88-0232, 1988. 
Public reporting burden for this collection of information is estimated to average 1 hour per response, including the time for reviewing instructions, searching existing data sources, gathering and maintaining the data needed, and completing and reviewing the collection of information. Send comments regarding this burden estimate or any other aspect of this collection of information, including suggestions for reducing this burden, to Washington Headquarters Services, Directorate for information Operations and Reports, 1215 Jefferson Davis Highway, Suite 1204, Arlington, VA 22202-4302, and to the Office of Management and Budget, Paperwork Reduction Project (0704-0188), Washington, DC 20503.

\begin{tabular}{|l|l|l}
\hline 1. AGENCY USE ONLY (Leave blank) & 2. REPORT DATE & $\begin{array}{r}\text { 3. REPORT TYPE AND DATES COVERED } \\
\text { Technical Memorandum }\end{array}$ \\
\hline
\end{tabular}

4. TITLE AND SUBTITLE

Progress Toward Synergistic Hypermixing Nozzles

6. AUTHOR(S)

D.O. Davis and W.R. Hingst

WU $-505-62-40$

\section{PERFORMING ORGANIZATION NAME(S) AND ADDRESS(ES)}

8. PERFORMING ORGANIZATION REPORT NUMBER

National Aeronautics and Space Administration

Lewis Research Center

Cleveland, Ohio 44135 - 3191

E- 6461

9. SPONSORING/MONITORING AGENCY NAMES(S) AND ADDRESS(ES)

National Aeronautics and Space Administration

Washington, D.C. 20546-0001
10. SPONSORING/MONITORING AGENCY REPORT NUMBER

NASA TM - 105169

AIAA - 91-2264

11. SUPPLEMENTARY NOTES

Prepared for the 27th Joint Propulsion Conference cosponsored by AIAA, SAE, ASME, and ASEE, Sacramento, California, June 24-27, 1991. Responsible person, D.O. Davis, (216) 433 - 8116.

12a. DISTRIBUTION/AVAILABILITY STATEMENT

12b. DISTRIBUTION CODE

Unclassified - Unlimited

Subject Category 02

\section{ABSTRACT (Maxlmum 200 words)}

Mean flow measurements have been obtained for air-to-air mixing downstream of swept and unswept ramp wall-mounted hypermixing nozzle configurations. Aside from the sweep of the ramps, the two nozzle configurations investigated are identical. The nozzles inject three parallel supersonic jets $\left(M_{j e t}=3.0\right)$ at a $15^{\circ}$ angle (relative to a wind tunnel wall) into a supersonic freestream. Mach number and volume fraction distributions in a transverse plane 11.1 nozzle heights downstream from the nozzle exit plane were measured. Data are presented for a freestream Mach number of three at a matched static pressure condition and also at an underexpanded static pressure condition (pressure ratio equal to five). Surface oil flow visualization was used to investigate the near-wall flow behavior. The results indicate that the swept ramp injectors produce stronger and larger vortex pairs than the unswept ramp injectors. The increased interaction between the swept ramp model's larger vortex pairs yields better mixing characteristics for this model.

14. SUBJECT TERMS

Turbulent mixing; Supersonic combustion ramjet engines; Supersonic nozzles; Supersonic jet flow

\begin{tabular}{|} 
15. NUMBER OF PAGES \\
10 \\
\hline $\begin{array}{c}\text { 16. PRICE CODE } \\
\mathrm{AO} 2\end{array}$ \\
\hline $\begin{array}{c}\text { 20. LIMITATION OF ABSTRACT } \\
\end{array}$
\end{tabular}

18. SECURITY CLASSIFICATION OF THIS PAGE Unclassified
19. SECURITY CLASSIFICATION OF ABSTRACT Unclassified 
National Aeronautics and Space Administration

Lewis Research Center

Cleveland, Ohio 44135

Official Business

Penalty for Private Use $\$ 300$
FOURTH CLASS MAIL.

ADDRESS CORRECTION REQUESTED

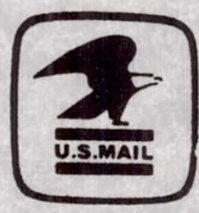

Posiage and Fees Paid

National Aeronautics and

Space Administration

NASA 451

\section{N/SA}

